\title{
A peroxiredoxin from Mycoplasma hyopneumoniae with a possible role in $\mathrm{H}_{2} \mathrm{O}_{2}$ detoxification
}

\section{Correspondence \\ Henrique B. Ferreira \\ henrique@cbiot.ufrgs.br}

Received 7 May 2009

Revised 30 June 2009

Accepted 6 July 2009

\author{
Cláudio X. Machado, ${ }^{1}$ Paulo M. Pinto, ${ }^{1}$ Arnaldo Zaha ${ }^{1,2}$ \\ and Henrique B. Ferreira ${ }^{1,2}$
${ }^{1}$ Laboratório de Genômica Estrutural e Funcional, Centro de Biotecnologia, UFRGS, Porto Alegre, RS, Brazil
${ }^{2}$ Departamento de Biologia Molecular e Biotecnologia, Instituto de Biociências, UFRGS, Porto Alegre-RS, Brazil

\begin{abstract}
Mycoplasma hyopneumoniae is the causative agent of porcine enzootic pneumonia, which affects pig farms worldwide, causing heavy economic losses. In the infection process, this bacterium is exposed to reactive oxygen species (ROS) from its own metabolism or generated by the host as one of the strategies used to neutralize the pathogen. Although the presence of classical antioxidant enzymes would be expected in M. hyopneumoniae, important genes directly related to protection against ROS, such as superoxide dismutase, catalases and glutathione peroxidase, have not been identified by sequence homology in the genome sequence annotation. Among the few identified $M$. hyopneumoniae genes coding for proteins possibly involved with suppression of ROS-mediated damage, one (tpx) coding for a peroxiredoxin (MhPrx) has been recognized. The sequence and phylogenetic analyses perfomed in this study indicate that MhPrx is closely related to the atypical 2-Cys peroxiredoxin subfamily, although it has only one cysteine in its sequence. The MhPrx coding DNA sequence was cloned and expressed in Escherichia coli to produce a recombinant MhPrx (rMhPrx), which was purified and used to immunize mice and produce an antiMhPrx polyclonal antiserum. Probing of $M$. hyopneumoniae extracts with this antiserum demonstrated that MhPrx is expressed in all three tested strains (J, 7422 and 7448). Cross-linking assays and size-exclusion chromatography indicate that $\mathrm{rMhPrx}$ forms dimers, as has been established for atypical 2-Cys peroxiredoxins. Furthermore, a metal-catalysed oxidation system was used to assay the activity of $\mathrm{rMhPrx}$, showing that it can protect DNA from ROS-mediated damage and may play an essential role during infection.
\end{abstract}

\section{INTRODUCTION}

Mycoplasma hyopneumoniae is the causative agent of porcine enzootic pneumonia (Mare \& Switzer, 1965), which affects pig farms worldwide. This highly contagious disease is characterized by a mild but chronic pneumonia that leads to low mortality but high morbidity (Messier et al., 1990). Along with growth retardation, and reduced feed conversion, the costs associated with currently available antibiotic treatments lead to heavy economic losses to swine production (Maes et al., 2008). In the infection process, the bacteria adhere to ciliated epithelial cells in the respiratory tract of the host, causing ciliostasis and loss of cilia (DeBey \& Ross, 1994). The damaged tracheal epithelium compromises the main defence of the host against aerosol-transmitted diseases and raises the risk and

Abbreviations: GST, glutathione S-transferase; MCO, metal-catalysed oxidation; ROS, reactive oxygen species.

A supplementary table and three supplementary figures are available with the online version of this paper. intensity of secondary infections by pathogens such as Pasteurella multocida (Ciprian et al., 1988) and the porcine reproductive and respiratory syndrome virus (Cho et al., 2006).

M. hyopneumoniae avoids swine defence mechanisms during early stages of pneumonia by inhibiting macrophage-mediated phagocytosis and B-cell antibody production (Caruso \& Ross, 1990; Ross, 1992). After the pathogen is established in the ciliated epithelium, lymphocytes infiltrate the site of infection, recruiting macrophages and neutrophils. Cellular immune responses in swine also include the production of reactive oxygen species (ROS), such as hydrogen peroxide $\left(\mathrm{H}_{2} \mathrm{O}_{2}\right)$ and superoxide anions $\left(\mathrm{O}_{2}^{-}\right)$, in a process known as the respiratory burst, which may kill an infective organism (du Manoir et al., 2002).

Apart from ROS produced by the host immune response, M. hyopneumoniae can be also exposed to $\mathrm{H}_{2} \mathrm{O}_{2}$ generated endogenously. Recent studies have demonstrated that other mycoplasmas can generate $\mathrm{H}_{2} \mathrm{O}_{2}$ or ROS as byproducts of 
glycerol metabolism, involving genes such as $g l p O$ or $g l p D$ (Pilo et al., 2005; Bischof et al., 2009; Hames et al., 2009). These metabolites can be translocated to the host cell, being involved in toxicity toward the host (Vilei \& Frey, 2001; Bischof et al., 2008). A $g l p D$ gene has been identified in the M. hyopneumoniae genome sequence and the corresponding protein has similarity levels of $64 \%$ and $63 \%$ to the well-characterized GlpD of Mycoplasma pneumoniae and GlpO of Mycoplasma mycoides subsp. mycoides SC, respectively.

On the other hand the M. hyopneumoniae genome lacks genes coding for some important antioxidant proteins, such as catalases, glutathione peroxidase and superoxide dismutase (Minion et al., 2004; Vasconcelos et al., 2005), and none of the at least four ROS-protection-related genes annotated in the M. hyopneumoniae genome (thiol peroxidase, peptide methionine sulfoxide reductase, thioredoxin, and thioredoxin reductase) was found to be transcriptionally upregulated under oxidative stress culture conditions (Schafer et al., 2007). However, since it is essential to M. hyopneumoniae to avoid ROS-mediated damage during infection, these observations are likely to be the consequence of evolutionary events, including loss of genes and/or divergence from common orthologues, and of a possible constitutive mode of expression for at least some of the antioxidant proteins available for this species.

Among the so far identified M. hyopneumoniae genes coding for putative ROS-protection proteins, one ( $t p x)$ coding for a peroxiredoxin was recognized by sequence homology and originally annotated as a probable thiol peroxidase (Vasconcelos et al., 2005). The peroxiredoxin superfamily comprises multifunctional thiol-dependent peroxidases that catalyse the reduction of various peroxide substrates, such as $\mathrm{H}_{2} \mathrm{O}_{2}$, peroxynitrite and organic hydroperoxides (Bryk et al., 2000; Castro et al., 2002; Knoops et al., 2007), and their basic mechanism relies on the oxidation of a peroxidatic cysteine residue in the active site to a sulfenic acid by the peroxide substrate (Rhee et al., 2005). The M. hyopneumoniae tpx gene codes for a 163 aa protein (MhPrx), with a single cysteine residue in the deduced sequence (Cys57). In this study, recombinant MhPrx (rMhPrx) was characterized and assayed for peroxidase activity. In vivo expression of MhPrx was analysed and the phylogenetic relationships of this putative alternative form of peroxiredoxin are discussed.

\section{METHODS}

M. hyopneumoniae strains, culture and protein extraction. $M$. hyopneumoniae non-pathogenic strain J (ATCC 25934) was acquired from the American Type Culture Collection by the EMBRAPACNPSA (Concórdia, Brazil). M. hyopneumoniae pathogenic strain 7448 was isolated from infected swine from Lindóia do Sul (Brazil) (Vasconcelos et al., 2005). M. hyopneumoniae pathogenic strain 7422, another Brazilian field isolate, was obtained from the EMBRAPACNPSA collection. M. hyopneumoniae cultivation and protein extraction were performed as described by Pinto et al. (2007).
Site-directed mutagenesis and cloning of the tpx gene. The complete 492 bp tpx coding DNA sequence (CDS) was obtained from an Escherichia coli pUC18 clone used in the genome sequencing of $M$. hyopneumoniae 7448 (Vasconcelos et al., 2005). A Trp-encoding TGA codon was mutated to TGG using the megaprimer PCR method (Angelaccio \& Bonaccorsi di Patti, 2002) with the following primers: 5'-TTAGGAAAATTTTTTTAGATTTTCTTC-3' (external reverse primer); 5'-GCCCAATGGAAAGATGCTAATTTAG-3' (mutagenic internal primer, with the mutated codon underlined); and 5'-ATGCAGACAAAATTTAAAAATAAATC-3' (external forward primer).

The mutated tpx CDS was cloned into the pGEX-4T-1 expression vector (GE Healthcare) for expression as a fusion with glutathione $S$ transferase (GST). The resultant pGEX-4T-1::tpx construct was confirmed by sequencing.

Recombinant MhPrx expression and purification. Fresh cultures of E. coli BL21 containing pGEX-4T-1::tpx were induced with $0.8 \mathrm{mM}$ IPTG at $28{ }^{\circ} \mathrm{C}$ for $16 \mathrm{~h}$. Cells were harvested, resuspended in PBS (10 mM Na $\mathrm{HPO}_{4}, 1.7 \mathrm{mM} \mathrm{K \textrm {H } _ { 2 }} \mathrm{PO}_{4}, 137 \mathrm{mM} \mathrm{NaCl}, 2.7 \mathrm{mM}$ $\mathrm{KCl}, \mathrm{pH} 7.4$ ) and sonicated at $75 \mathrm{~Hz}$ in an ice bath. Triton $\mathrm{X}-100$ (Sigma) was added to a final concentration of $2 \%$ and the cell extract was centrifuged at $13000 \mathrm{~g}$ for $30 \mathrm{~min}$. Purification was performed in a Glutathione Sepharose 4B (GE Healthcare) affinity column, following the manufacturer's recommendations. The rMhPrx was recovered free of the GST tag after on-column proteolytic cleavage with thrombin (Sigma) at $22{ }^{\circ} \mathrm{C}$ for $16 \mathrm{~h}$. The concentration of rMhPrx was determined by SDS-PAGE analysis of serial dilutions compared to known quantities of BSA using a GS-800 densitometer (Bio-Rad) and the Quantity One v. 4.6.3 software (Bio-Rad).

Size exclusion chromatography. A $500 \mu \mathrm{g}$ sample of rMhPrx in PBS was loaded onto a Superdex 75 HR 10/30 (separation range: 3000-70 $000 \mathrm{Da}$ ) column (Pharmacia Biotech) previously equilibrated in PBS. Chromatography was performed on a Pharmacia FPLC system (Pharmacia Biotech) at a flow rate of $0.5 \mathrm{ml} \mathrm{min}^{-1}$ at room temperature and the absorbance was monitored at $280 \mathrm{~nm}$. The column was calibrated using protein molecular mass standards under the same conditions.

Cross-linking assay. Cross-linking assays were carried out as described by Monteiro et al. (2007b). In brief, $80 \mu \mathrm{l}$ of a $3 \mathrm{mg}$ $\mathrm{ml}^{-1}$ solution of $\mathrm{rMhPrx}$ in PBS was incubated with $0.1 \%(\mathrm{v} / \mathrm{v})$ glutaraldehyde at room temperature. Ten-microlitre aliquots were collected at different times and reactions were stopped by adding SDS sample buffer and boiling at $100{ }^{\circ} \mathrm{C}$ for $5 \mathrm{~min}$. Cross-linked products were analysed under reducing conditions by $15 \%$ SDS-PAGE.

In vitro peroxidase activity assay. For each batch of purified rMhPrx, peroxidase activity was assessed in triplicate using a metalcatalysed oxidation (MCO) system ( $\mathrm{Li}$ et al., 2004), with some modifications. Reaction mixtures of $20 \mu \mathrm{l}$ contained $150 \mu \mathrm{M} \mathrm{FeCl}_{3}$, $10 \mathrm{mM}$ DTT, $0.1 \mu \mathrm{g}$ pUC18 supercoiled DNA $\mu \mathrm{l}^{-1}, 0.1 \mathrm{mM}$ EDTA and $25 \mathrm{mM}$ Tris $\mathrm{pH}$ 7.5. DNA nicking was assayed in the absence or presence of two concentrations $(3.75 \mu \mathrm{M}$ and $7.5 \mu \mathrm{M})$ of rMhPrx, in the presence of $7.5 \mu \mathrm{M}$ GST (negative control) and in the absence of $\mathrm{Fe}^{3+}$ (positive control). Fractions of $5 \mu \mathrm{l}$ were collected at different times, inactivated with EDTA to a final concentration of $50 \mathrm{mM}$ and electrophoretically analysed. Gels were photographed with Gel Doc XR (Bio-Rad), and the Quantity One v. 4.6.3 software (Bio-Rad) was used to estimate the relative concentrations of the observed bands. The percentage of plasmid supercoiled DNA remaining in each treatment after $2 \mathrm{~h}$ of incubation was compared using one-way ANOVA followed by Tukey's multiple comparison tests at a $5 \%$ significance level. All statistic analyses were performed with SPSS v. 14.0 (http://www.spss.com/). 
Western blotting. Antiserum was produced by immunizing three 60 -day-old BALB/c mice with four doses of $20 \mu \mathrm{g} \mathrm{rMhPrx}$, at 14 day intervals. Individual sera were pooled to produce the anti-MhPrx serum used in the experiments. All procedures and experiments involving animals were performed according to Brazilian laws and were approved by the Ethical Committee of the Universidade Federal do Rio Grande do Sul.

M. hyopneumoniae total protein extracts $(80 \mu \mathrm{g}$ per lane $)$ were fractionated by $15 \%$ SDS-PAGE and then transferred to nitrocellulose membranes (Amersham Biosciences). Membranes were blocked with $5 \%(\mathrm{w} / \mathrm{v})$ skim milk powder in PBS (blotto-PBS) and incubated with anti-MhPrx serum or pre-immune serum. Antimouse IgG peroxidaseconjugated antibody (Sigma) served as the secondary antibody and reactions were visualized with the ECL Plus kit (GE Healthcare).

Sequence and phylogenetic analyses. The NCBI BLAST tool (http://www.ncbi.nlm.nih.gov) was used to search for orthologues of the MhPrx deduced amino acid sequence in the updated GenBank/ EMBL and Swiss-Prot databases. Multiple sequence alignments of MhPrx and other peroxiredoxins were performed with the Muscle v. 3.6 software (http://www.genebee.msu.su) and edited using GenDoc v. 2.7.0 (http://www.nrbsc.org/gfx/genedoc/). Phylogenetic relationships were determined using the neighbour-joining (NJ) method and MEGA v. 4.0 (Tamura et al., 2007).

\section{RESULTS}

\section{Recombinant MhPrx production}

The rMhPrx was overexpressed in E. coli and purified to homogeneity free of the GST tag. After cleavage with thrombin, the sequence of the resulting peptide was the same as the deduced native protein sequence plus a GSPELGTL amino acid extension appended to its $\mathrm{N}$-terminus, resulting from the cloning procedure. On SDS-PAGE analysis (Fig. la, lane rMhPrx), a single band was observed with a molecular mass of approximately $18.5 \mathrm{kDa}$, as expected.

\section{Recombinant MhPrx dimerization}

The dimerization of rMhPrx was first tested by glutaraldehyde cross-linking experiments. A band with the apparent molecular mass of a MhPrx dimer was visualized with a concentration of $0.1 \%$ glutaraldehyde from $0.5 \mathrm{~min}$ incubation and this association was further stabilized with longer incubation times (Fig. 1a). In a second approach, size-exclusion chromatography was used to examine the apparent molecular mass of rMhPrx in non-denaturing conditions. The elution profile revealed a major peak with the apparent molecular mass of a MhPrx monomer and a secondary peak with the apparent molecular mass of a MhPrx dimer (Fig. 1b). SDS-PAGE analysis confirmed the presence of the $18.5 \mathrm{kDa}$ rMhPrx in all fractions collected from both peaks (data not shown).

\section{Peroxidase activity of $\mathrm{rMhPrx}$}

An MCO system was used to evaluate whether rMhPrx can protect supercoiled DNA from degradation. The extent of DNA damage was evaluated by the relative quantification
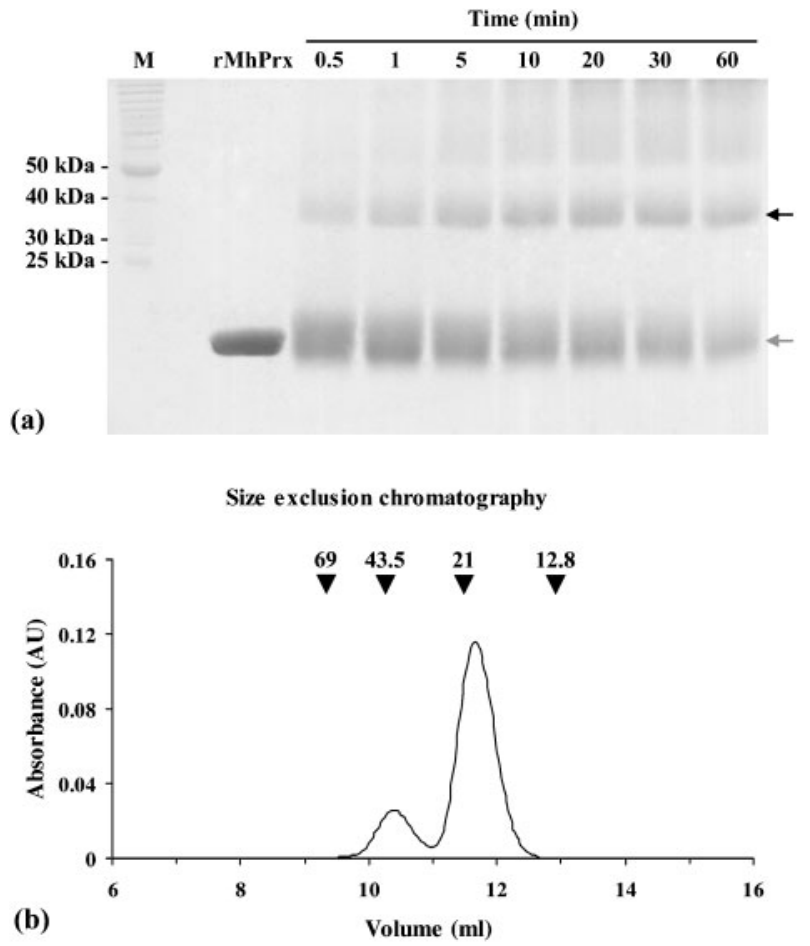

Fig. 1. Dimerization of rMhPrx. (a) rMhPrx was cross-linked with $0.1 \%$ glutaraldehyde for $0.5,1,5,10,20,30$ and $60 \mathrm{~min}$, as indicated (top), and separated by $15 \%$ SDS-PAGE followed by Coomassie blue staining. The grey arrow indicates the expected molecular mass of MhPrx monomer $(18.5 \mathrm{kDa})$. The black arrow indicates the cross-linked products of approximately $37 \mathrm{kDa}$, the expected molecular mass of the dimeric form of MhPrx. The relevant molecular mass standards are indicated (lane $M$ ). The purified recombinant MhPrx (rMhPrx) was also included for reference. (b) Elution profile of $\mathrm{rMhPrx}$ chromatographed on a Superdex 75 HR 10/30 column. BSA (69 kDa), ovalbumin $(43.5 \mathrm{kDa})$, soybean trypsin inhibitor $(21 \mathrm{kDa})$ and cytochrome $c$ $(12.8 \mathrm{kDa})$ were used as molecular mass standards and their positions are indicated by arrowheads (top).

of electrophoretically resolved pUC18 supercoiled and nicked (linear) forms in each sample. Results obtained with a representative batch of purified rMhPrx are presented in Fig. 2 and Supplementary Table S1 (available with the online version of this paper). Fig. 2(b) shows the percentage of supercoiled DNA remaining after $2 \mathrm{~h}$ of incubation. In the absence of $\mathrm{Fe}^{3+}$, no nicking of plasmid DNA was observed in comparison to plasmid incubated in water, whereas the same reaction including $\mathrm{Fe}^{3+}$ caused a statistically significant decrease of the band corresponding to pUC18 supercoiled DNA. The addition of rMhPrx at two concentrations $(3.75 \mu \mathrm{M}$ and $7.5 \mu \mathrm{M})$ prevented nicking of the supercoiled DNA in the assay in comparison with the plasmid incubated with the MCO system, but with no significant difference between the two concentrations of enzyme. No protection of plasmid DNA was observed with $7.5 \mu \mathrm{M}$ GST. The experiment was repeated for different 

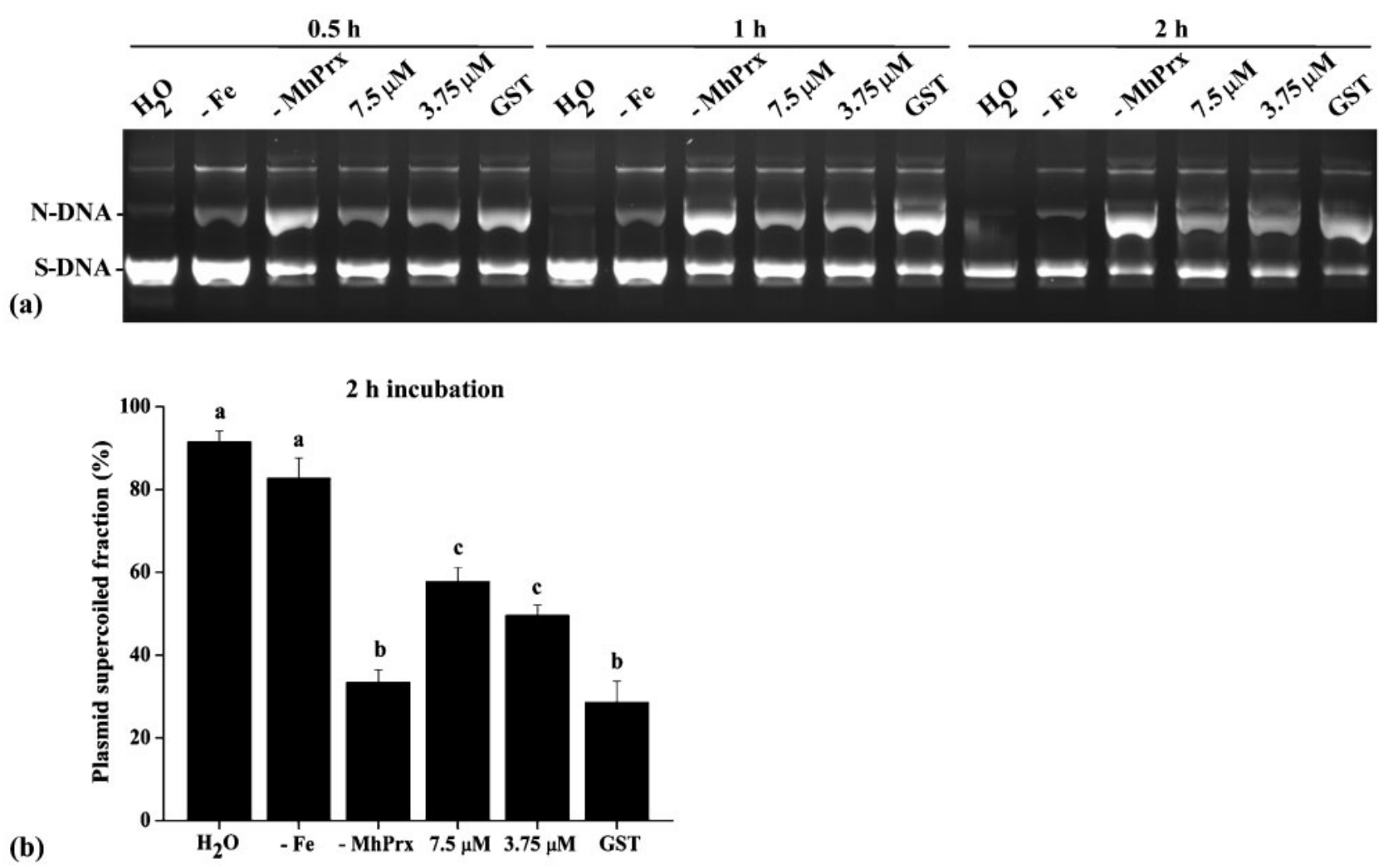

(b)

Fig. 2. Peroxidase activity of $r M h P r x$ assayed in an MCO system. (a) Fractions were collected from the different treatment tubes and electrophoretically analysed in $1 \%$ agarose gel. The nicked form (N-DNA) and the parental supercoiled form (S-DNA) of pUC18 are indicated. Fractions are from incubation of pUC18 in water $\left(\mathrm{H}_{2} \mathrm{O}\right)$, in the $\mathrm{MCO}$ system without $\mathrm{FeCl}_{3}$ (positive control; -Fe), and in the MCO system without rMhPrx (negative control; -MhPrx) or in the presence of two different concentrations of $\mathrm{rMhPrx}(7.5 \mu \mathrm{M}$ and $3.75 \mu \mathrm{M})$; purified GST (7.5 $\mu \mathrm{M}$; GST) was also used in place of MhPrx as a negative control. Incubation time and the different conditions are shown above each lane. (b) Fraction of supercoiled DNA remaining after $2 \mathrm{~h}$ of incubation. Treatments with no statistically significant difference (at a $5 \%$ level) in Tukey's multiple comparisons tests are grouped with the letters $a, b$ and c. The percentage values represent the relative intensity of the supercoiled band, in comparison to the sum of intensities of the supercoiled band and the nicked band, for each treatment. Intensities were measured by densitometry from three repeats using a single batch of purified rMhPrx. Standard deviations are shown.

batches of purified rMhPrx, always with equivalent results (data not shown).

\section{Demonstration of MhPrx expression in different M. hyopneumoniae strains}

The murine anti-MhPrx serum was used in Western blot experiments to probe protein extracts of three different $M$. hyopneumoniae strains (J, 7422 and 7448). As shown in Supplementary Fig. S1, a band between 15 and $20 \mathrm{kDa}$, in agreement with the expected molecular mass of the monomeric form of MhPrx (18.5 kDa), was detected in all strains, but was absent in replicate membranes probed with the pre-immune serum.

\section{Sequence analysis of MhPrx}

MhPrx was regarded as a 1-Cys peroxiredoxin, since it has a single cysteine residue. However, despite the lack of a second cysteine residue (the so-called resolving cysteine), a BLAST search against the NCBI databases using the MhPrx deduced amino acid sequence as query sequence (data not shown) returned specific hits and detected putative conserved domains only with the atypical 2-Cys peroxiredoxin subfamily, with no significant similarity to 1-Cys peroxiredoxin subfamily members. Sequence alignment of MhPrx and nine bacterial atypical 2-Cys peroxiredoxins (Fig. 3) revealed that the M. hyopneumoniae enzyme shows conserved amino acid residues with these proteins throughout its sequence, with overall identities and similarities ranging between $18 \%$ and $33 \%$, and $36 \%$ and $51 \%$, respectively. The catalytic triad is $100 \%$ conserved and five out of eight residues of the putative dimerization domain are conserved (considering their physicochemical properties), whereas the other three residues do not show similarity with the subfamily members, although they are less conserved. The MhPrx 

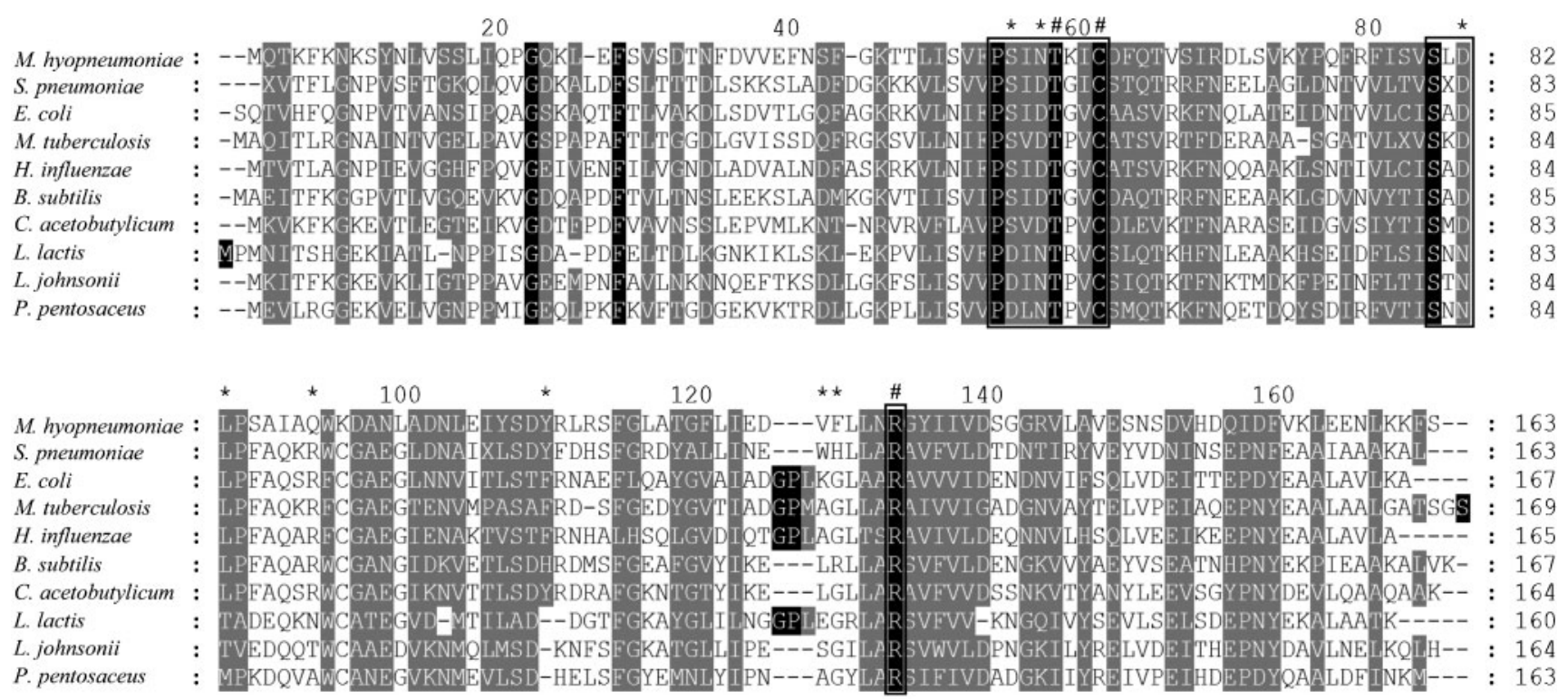

Fig. 3. Multiple sequence alignment of MhPrx and nine bacterial members of the atypical 2-Cys peroxiredoxin subfamily. Identical and similar amino acids (considering their physico-chemical properties) are denoted by black and grey backgrounds, respectively. Black rectangles indicate residues spatially related to the catalytic site, including the peroxidatic cysteine at position 57 in MhPrx. Residues constituting the catalytic triad (\#) and the putative dimer interface $\left(^{*}\right)$ are indicated. Organisms from which sequences were taken (and sequence accession numbers) are: Streptoccoccus pneumoniae (YP_816920), Escherichia coli (NP_287864), Mycobacterium tuberculosis (1XVQ A), Haemophilus influenzae (NP_438910), Bacillus subtilis (NP_390827), Clostridium acetobutylicum (NP_349898), Lactococcus lactis (NP_266460), Lactococcus johnsonii (NP_965009) and Pediococcus pentosaceus (YP_804775).

sequence was also aligned with nine bacterial 1-Cys peroxiredoxins (Supplementary Fig. S2), revealing low overall scores of similarity and identity (in the range from $3 \%$ to $9 \%$ and from $24 \%$ to $27 \%$, respectively). As expected, given that part of the catalytic mechanism is shared by all peroxiredoxins, there is some conservation in residues of the active site.

\section{Phylogenetic analysis of MhPrx}

To further investigate the evolutionary relationships of mycoplasmal peroxiredoxins with other bacterial orthologues, a phylogenetic tree was constructed. MhPrx and another three mycoplasmal peroxiredoxins (from $\mathrm{Myco}$ plasma synoviae, Mycoplasma mobile and Mycoplasma pulmonis) were aligned to known atypical 2-Cys or AhpE-like peroxiredoxins from 25 bacterial species, with the atypical 2-Cys peroxiredoxin from Saccharomyces cerevisiae, Dot5p, as an out-group (Fig. 4a). Atypical 2Cys and AhpE-like peroxiredoxins grouped separately, as expected, supported by a bootstrap value of $99 \%$. MhPrx, together with the other mycoplasmal peroxiredoxins, grouped with the known atypical 2-Cys clade rather than with the AhpE-like clade.

An additional analysis was conducted within the Mycoplasma clade using peroxiredoxins from seven differ- ent species (the four previously used plus those of Mycoplasma agalactiae, Mycoplasma capricolum subsp. capricolum and Mycoplasma penetrans), with the Acholeplasma laidlawii peroxiredoxin as out-group (Fig. 4b). This analysis divided the Mycoplasma species into two groups (one containing $M$. hyopneumoniae, M. synoviae, $M$. mobile and $M$. pulmonis, and the other one containing $M$. agalactiae, $M$. capricolum subsp. capricolum and $M$. penetrans) and showed that the closest sequence to MhPrx is that from M. synoviae peroxiredoxin.

\section{DISCUSSION}

In the present study we demonstrated that MhPrx has a DNA-protecting peroxidase activity and that it is expressed in both non-pathogenic ( $\mathrm{J}$ ) and pathogenic (7422 and 7448) M. hyopneumoniae strains. Swine can generate ROS both by means of their immune response and as byproducts of respiration, $\mathrm{H}_{2} \mathrm{O}_{2}$ and $\mathrm{O}_{2}^{-}$being commonly present in the host environment (du Manoir et al., 2002). It has also been demonstrated that mycoplasmas themselves produce $\mathrm{H}_{2} \mathrm{O}_{2}$ as byproducts of glycerol metabolism, this toxic metabolite being involved in virulence in species such as M. mycoides subsp. mycoides SC and M. pneumoniae (Bischof et al., 2008, Hames et al., 2009). Moreover, despite the absence of a known gene coding for superoxide 
(a)

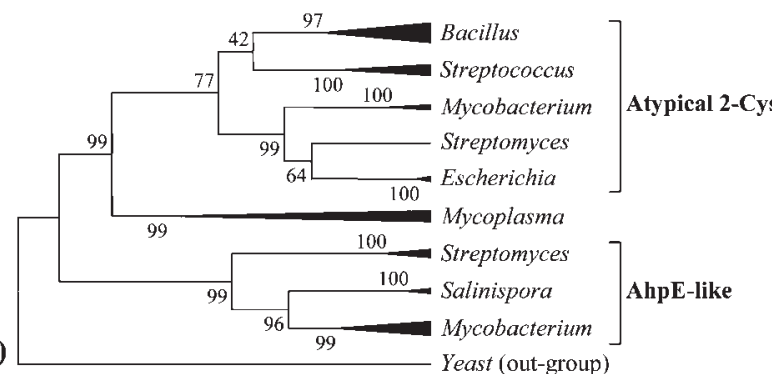

(b)

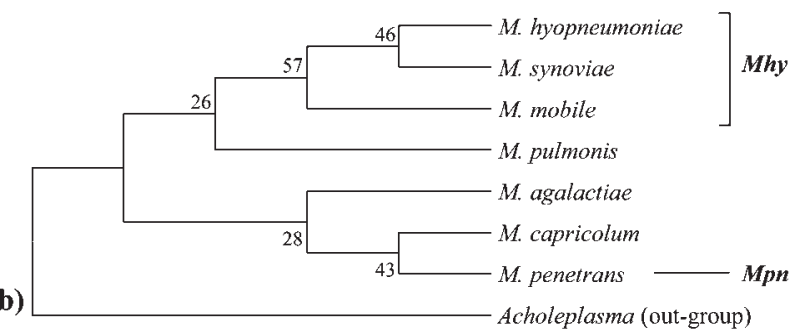

Fig. 4. Phylogenetic relationships of MhPrx. (a) Neighbour-joining tree of aligned peroxiredoxins of four Mycoplasma species ( $M$. hyopneumoniae, M. synoviae, M. mobile and M. pulmonis) and another 25 bacterial species from six genera, with Saccharomyces cerevisiae Dot5p (atypical 2-Cys peroxiredoxin, accession number NP_012255) as an out-group. Organisms from which sequences were taken (and sequence accession numbers) are as follows. Atypical 2-Cys - Bacillus: B. licheniformis (YP_080231), B. pumilus (ZP_03055981), B. amyloliquefaciens (YP_001422231), $B$. cereus (ZP_03108109), B. thuringiensis (ZP_00740038), B. selenitireducens (ZP_02170589), B. clausii (YP_176238); Streptococcus: S. agalactiae (NP_688140), S. pneumoniae (ZP_01408570), S. gordonii (YP_001451072); Mycobacterium: M. tuberculosis (1XVQ A), M. ulcerans (YP_906643); Streptomyces clavuligerus (ZP_03181049); Escherichia: E. albertii (ZP_02903301), E. coli (NP_287864). AhpE-like Streptomyces: S. sviceus (YP_002203881), S. coelicolor (NP_626616), S. griseus (YP_001826644); Salinispora: S. arenicola (YP_001538397), $\bar{S}$. tropica (YP_001160179); Mycobacterium: M. vanbaalenii (YP_954537), M. gilvum (YP_001134049), M. avium (NP_960925), M. tuberculosis (1XV̄W A), M. ulcerans (YP_905342). (b) Neighbour-joining tree of seven aligned peroxiredoxins from the Mycoplasma clade, with the peroxiredoxin of Acholeplasma laidlawii, one of the first mollicutes to evolve by genome reduction (Razin et al., 1998; Sirand-Pugnet et al., 2007), as an out-group. Species representing the Mhy and Mpn clusters, defined by Oshima \& Nishida (2007), are indicated. Organisms from which sequences were taken (and sequence accession numbers) are: M. hyopneumoniae (YP_287496), M. synoviae (YP_278491), M. mobile (YP_015976), M. pulmonis (NP_326539), M. agalactiae (YP_001256344), M. capricolum subsp. capricolum (YP_424787), M. penetrans (NP_757783), Acholeplasma laidlawii (YP_001620344). Bootstrap test values (1000 replicates) are shown. dismutase (SOD) in its genome (Vasconcelos et al., 2005), a $\mathrm{Cu} / \mathrm{ZnSOD}$ activity has been demonstrated in $M$. hyopneumoniae (Chen et al., 2000), catalysing the dismutation of $\mathrm{O}_{2}^{-}$and contributing to the formation of $\mathrm{H}_{2} \mathrm{O}_{2}$. MhPrx is the only $\mathrm{H}_{2} \mathrm{O}_{2}$-metabolizing enzyme identified in M. hyopneumoniae so far, and it is expected to play a major role in detoxification of $\mathrm{H}_{2} \mathrm{O}_{2}$ resulting from all the abovementioned sources.

Our in vitro results with rMhPrx indicate that it exists in part as a dimer. Given its similarity to members of the atypical 2-Cys peroxiredoxin subfamily, it is probable that the native form of MhPrx is actually a dimer. It has been established that atypical 2-Cys peroxiredoxins are active as dimers and eight amino acid residues are proposed to form the dimer interface in members of this subfamily (Choi et al., 2003; Wood et al., 2003; Rho et al., 2006). In MhPrx, there is a partial conservation of the putative dimer interface, and homodimerization probably involves these amino acid residues.

The MCO system has been widely used to assess ROS damage to DNA and other biomolecules such as proteins and lipids (Rho et al., 2006; Pushpamali et al., 2008; Suttiprapa et al., 2008) and has been previously used to assay the activity of peroxiredoxins from different subfamilies (Li et al., 2004; Rho et al., 2006; Wang et al., 2008). The $\mathrm{Fe}^{3+}$ present in the MCO system reacts with $\mathrm{O}_{2}$, generating the intermediate product $\mathrm{H}_{2} \mathrm{O}_{2}$ and ultimately hydroxyl radicals $\left(\mathrm{OH}^{-}\right)$that can nick supercoiled plasmid DNA into a linear form. Our results using the MCO system showed that MhPrx has a peroxidase activity acting on the $\mathrm{H}_{2} \mathrm{O}_{2}$ intermediate and protecting plasmid DNA from nicking. Despite the apparent difference between the treatments with $7.5 \mu \mathrm{M}$ and $3.75 \mu \mathrm{M}$ MhPrx, the difference was not significant using post hoc analysis with Tukey's multiple comparison tests. However, the use of other less stringent ANOVA post hoc tests resulted in a significant difference between the two treatments (data not shown); this lack of a dose-dependence in our experiments is likely to be a consequence of the stringency of the Tukey's multiple comparison tests.

During the catalytic cycle of peroxiredoxins, the peroxidatic cysteine is first oxidized to a sulfenic acid and is reduced in a second step. Assignment of enzyme names depends on the nature of the reducing agent. In the MCO system assay, the reducing agent (DTT) is artificial; the natural reducing agent for MhPrx remains to be identified. MhPrx was originally described as a thiol peroxidase (Vasconcelos et al., 2005), but, since its mechanism of action has not been fully elucidated and at least some 1-Cys peroxiredoxins can use non-thiolic electron donors to reduce the peroxidatic cysteine sulfenic acid intermediate (Monteiro et al., 2007a), we decided to treat this enzyme generically as a peroxiredoxin.

MhPrx expression in M. hyopneumoniae 7448 has been previously reported based on proteomic studies (Pinto et al., 2007), where it appears as a strong spot even in 
Coomassie blue stained 2D SDS-PAGE. Here, we demonstrate that MhPrx is also expressed in the J and 7422 strains, in accordance with our unpublished proteomic results. Microarray-based global transcription analyses have not detected any changes in the expression level of the tpx gene in the M. hyopneumoniae 232 after exposure to $\mathrm{H}_{2} \mathrm{O}_{2}$ (Schafer et al., 2007). Thus, the expression of MhPrx in three different strains even under culture conditions may suggest additional roles for this protein in the biology of $M$. hyopneumoniae, resulting in a constitutive mode of expression. Another possible explanation could be the expression of MhPrx in response to endogenous oxidative stress, which is supported by the presence of genes (such as $g l p D)$ that participate in ROS-generating metabolic pathways in this bacterium. High levels of expression of peroxiredoxins have also been reported for E. coli, where they are among the ten most expressed proteins (Link et al., 1997), and for mammalian cells, in which peroxiredoxins represent $0.1-0.8 \%$ of soluble proteins (Seo et al., 2000) under standard culture conditions and not only under induced oxidative stress.

Peroxiredoxin classification was originally proposed based on the number of cysteine residues present in the protein sequence involved in the enzymic mechanism and in the conservation of residues around the catalytic cysteines (Rhee et al., 2005). According to this classification, peroxiredoxins can be divided into two subfamilies, the 1-Cys and the 2-Cys. The 2-Cys peroxiredoxins are further divided into typical or atypical based on the formation of an intermolecular or an intramolecular disulfide bond in the catalytic cycle, respectively. In our phylogenetic analysis, MhPrx grouped, along with the other three mycoplasmal peroxiredoxins (from M. synoviae, M. mobile and $M$. pulmonis) within the atypical 2-Cys subfamily. Accordingly, M. mobile and M. pulmonis have two cysteines in their amino acid sequences matching the conserved peroxidatic and resolving cysteines present in atypical 2Cys peroxiredoxins. M. agalactiae, M. capricolum subsp. capricolum and $M$. penetrans peroxiredoxins were excluded from this first analysis because they did not group within any clade, but they also have two cysteines in their sequences. However, M. hyopneumoniae and M. synoviae peroxiredoxins have only the peroxidatic cysteine, and lack the resolving cysteine. A 1-Cys peroxiredoxin with structure and sequence showing more similarity to 2-Cys peroxiredoxins was reported in Mycobacterium tuberculosis (AhpE) (Li et al., 2005) and a number of orthologues found in other organisms (Stinear et al., 2007; Ohnishi et al., 2008) have been grouped with it into a new subfamily, namely AhpE-like. However, in our phylogenetic tree, mycoplasmal peroxiredoxins did not group with the AhpElike peroxiredoxins, being more closely related to atypical 2-Cys subfamily members.

M. hyopneumoniae and M. synoviae, along with M. mobile and M. pulmonis, are members of the M. hyopneumoniae $(M h y)$ group, as proposed by whole-genome sequence analysis (Oshima \& Nishida, 2007). On the other hand, $M$. penetrans is proposed to be a member of the $M$. pneumoniae ( $M p n)$ group, whereas M. agalactiae and $M$. capricolum subsp. capricolum were excluded from the abovementioned analysis. Despite its low bootstrap values, our mycoplasmal peroxiredoxin phylogenetic tree showed an overall arrangement that agrees with the classification described by Oshima \& Nishida (2007), separating $M$. penetrans (Mpn group) from the Mhy group. Altogether, these data suggest that, within the Mhy group, as in $M$. hyopneumoniae and M. synoviae, an atypical 2-Cys peroxiredoxin may have evolved to a 1-Cys peroxiredoxin by losing the resolving cysteine and keeping the peroxidatic cysteine. The resultant peroxiredoxin is still functional, as indicated by our rMhPrx in vitro assays, but its catalytic mechanism may differ from that of atypical 2-Cys peroxiredoxins and remains to be elucidated.

Further studies will be necessary to demonstrate MhPrx activity in vivo and to investigate its specific roles in $M$. hyopneumoniae. It is likely that its primary function is detoxification of $\mathrm{H}_{2} \mathrm{O}_{2}$, whether generated endogenously or in the natural environment, and that its role is essential for $M$. hyopneumoniae survival. This, associated with the considerable divergence of MhPrx and mammalian peroxiredoxins, makes this enzyme an attractive target to screen for inhibitors that could be used to develop drugs against porcine enzootic pneumonia. Currently, there are no specific inhibitors of the peroxiredoxin family of enzymes (Li et al., 2004). Apart from being one of the few proteins related to ROS protection identified in M. hyopneumoniae to date and the first one to be functionally characterized, MhPrx turns out to be also extremely interesting from the evolutionary point of view. It is representative of a possible group of atypical 2-Cys peroxiredoxins which have lost the resolving cysteine residue without losing their catalytic properties, and the future elucidation of its mechanism of action may enable a better understanding of the evolution of the peroxiredoxin superfamily.

\section{ACKNOWLEDGEMENTS}

We thank M.Sc. L. C. T. Silveira and R. X. Cortes for their help with the phylogenetic and statistical analyses, respectively. We also thank Dr C. R. Carlini and Dr D. Olivera-Severo for assistance with the FPLC system. C. X. M. was a recipient of a CNPq M.Sc. fellowship. P. M. P. was a recipient of a CNPq predoctoral fellowship. This work was supported by grants from the Brazilian science funding agencies $\mathrm{MCT} / \mathrm{CNPq}$ and FAPERGS.

\section{REFERENCES}

Angelaccio, S. \& Bonaccorsi di Patti, M. C. (2002). Site-directed mutagenesis by the megaprimer PCR method: variations on a theme for simultaneous introduction of multiple mutations. Anal Biochem 306, 346-349.

Bischof, D. F., Janis, C., Vilei, E. M., Bertoni, G. \& Frey, J. (2008). Cytotoxicity of Mycoplasma mycoides subsp. mycoides small colony type to bovine epithelial cells. Infect Immun 76, 263-269. 
Bischof, D. F., Vilei, E. M. \& Frey, J. (2009). Functional and antigenic properties of GlpO from Mycoplasma mycoides subsp. mycoides SC: characterization of a flavin adenine dinucleotide-binding site deletion mutant. Vet Res 40, 35.

Bryk, R., Griffin, P. \& Nathan, C. (2000). Peroxynitrite reductase activity of bacterial peroxiredoxins. Nature 407, 211-215.

Caruso, J. P. \& Ross, R. F. (1990). Effects of Mycoplasma hyopneumoniae and Actinobacillus (Haemophilus) pleuropneumoniae infections on alveolar macrophage functions in swine. Am J Vet Res 51, 227-231.

Castro, H., Budde, H., Flohé, L., Hofmann, B., Lünsdorf, H., Wissing, J. \& Tomás, A. M. (2002). Specificity and kinetics of a mitochondrial peroxiredoxin of Leishmania infantum. Free Radic Biol Med 33, 1563-1574.

Chen, J. R., Weng, C. N., Ho, T. Y., Cheng, I. C. \& Lai, S. S. (2000). Identification of the copper-zinc superoxide dismutase activity in Mycoplasma hyopneumoniae. Vet Microbiol 73, 301-310.

Cho, J. G., Dee, S. A., Deen, J., Guedes, A., Trincado, C., Fano, E., Jiang, Y., Faaberg, K., Collins, J. E. \& other authors (2006). Evaluation of the effects of animal age, concurrent bacterial infection, and pathogenicity of porcine reproductive and respiratory syndrome virus on virus concentration in pigs. Am J Vet Res 67, 489493.

Choi, J., Choi, S., Choi, J., Cha, M. K., Kim, I. H. \& Shin, W. (2003). Crystal structure of Escherichia coli thiol peroxidase in the oxidized state: insights into intramolecular disulfide formation and substrate binding in atypical 2-Cys peroxiredoxins. J Biol Chem 278, 4947849486.

Ciprian, A., Pijoan, C., Cruz, T., Camacho, J., Tortora, J., Colmenares, G., Lopez-Revilla, R. \& de la Garza, M. (1988). Mycoplasma hyopneumoniae increases the susceptibility of pigs to experimental Pasteurella multocida pneumonia. Can J Vet Res 52, 434-438.

DeBey, M. C. \& Ross, R. F. (1994). Ciliostasis and loss of cilia induced by Mycoplasma hyopneumoniae in porcine tracheal organ cultures. Infect Immun 62, 5312-5318.

du Manoir, J. M., Albright, B. N., Stevenson, G., Thompson, S. H., Mitchell, G. B., Clark, M. E. \& Caswell, J. L. (2002). Variability of neutrophil and pulmonary alveolar macrophage function in swine. Vet Immunol Immunopathol 89, 175-186.

Hames, C., Halbedel, S., Hoppert, M., Frey, J. \& Stulke, J. (2009). Glycerol metabolism is important for cytotoxicity of Mycoplasma pneumoniae. J Bacteriol 191, 747-753.

Knoops, B., Loumaye, E. \& Van Der Eecken, V. (2007). Evolution of the peroxiredoxins. Subcell Biochem 44, 27-40.

Li, J., Zhang, W. B., Loukas, A., Lin, R. Y., Ito, A., Zhang, L. H., Jones, M. \& McManus, D. P. (2004). Functional expression and characterization of Echinococcus granulosus thioredoxin peroxidase suggests a role in protection against oxidative damage. Gene 326, 157-165.

Li, S., Peterson, N. A., Kim, M. Y., Kim, C. Y., Hung, L. W., Yu, M., Lekin, T., Segelke, B. W., Lott, J. S. \& Baker, E. N. (2005). Crystal structure of AhpE from Mycobacterium tuberculosis, a 1-Cys peroxiredoxin. J Mol Biol 346, 1035-1046.

Link, A. J., Robison, K. \& Church, G. M. (1997). Comparing the predicted and observed properties of proteins encoded in the genome of Escherichia coli K-12. Electrophoresis 18, 1259-1313.

Maes, D., Segales, J., Meyns, T., Sibila, M., Pieters, M. \& Haesebrouck, F. (2008). Control of Mycoplasma hyopneumoniae infections in pigs. Vet Microbiol 126, 297-309.

Mare, C. J. \& Switzer, W. P. (1965). New species: Mycoplasma hyopneumoniae; a causative agent of virus pig pneumonia. Vet Med Small Anim Clin 60, 841-846.
Messier, S., Ross, R. F. \& Paul, P. S. (1990). Humoral and cellular immune responses of pigs inoculated with Mycoplasma hyopneumoniae. Am J Vet Res 51, 52-58.

Minion, F. C., Lefkowitz, E. J., Madsen, M. L., Cleary, B. J., Swartzell, S. M. \& Mahairas, G. G. (2004). The genome sequence of Mycoplasma hyopneumoniae strain 232, the agent of swine mycoplasmosis. J Bacteriol 186, 7123-7133.

Monteiro, G., Horta, B. B., Pimenta, D. C., Augusto, O. \& Netto, L. E. (2007a). Reduction of 1-Cys peroxiredoxins by ascorbate changes the thiol-specific antioxidant paradigm, revealing another function of vitamin C. Proc Natl Acad Sci U S A 104, 4886-4891.

Monteiro, K. M., Scapin, S. M., Navarro, M. V., Zanchin, N. I., Cardoso, M. B., da Silveira, N. P., Goncalves, P. F., Stassen, H. K., Zaha, A. \& Ferreira, H. B. (2007b). Self-assembly and structural characterization of Echinococcus granulosus antigen B recombinant subunit oligomers. Biochim Biophys Acta 1774, 278-285.

Ohnishi, Y., Ishikawa, J., Hara, H., Suzuki, H., Ikenoya, M., Ikeda, H., Yamashita, A., Hattori, M. \& Horinouchi, S. (2008). Genome sequence of the streptomycin-producing microorganism Streptomyces griseus IFO 13350. J Bacteriol 190, 4050-4060.

Oshima, K. \& Nishida, H. (2007). Phylogenetic relationships among mycoplasmas based on the whole genomic information. J Mol Evol 65, 249-258.

Pilo, P., Vilei, E. M., Peterhans, E., Bonvin-Klotz, L., Stoffel, M. H., Dobbelaere, D. \& Frey, J. (2005). A metabolic enzyme as a primary virulence factor of Mycoplasma mycoides subsp. mycoides small colony. J Bacteriol 187, 6824-6831.

Pinto, P. M., Chemale, G., de Castro, L. A., Costa, A. P., Kich, J. D., Vainstein, M. H., Zaha, A. \& Ferreira, H. B. (2007). Proteomic survey of the pathogenic Mycoplasma hyopneumoniae strain 7448 and identification of novel post-translationally modified and antigenic proteins. Vet Microbiol 121, 83-93.

Pushpamali, W. A., de Zoysa, M., Kang, H. S., Oh, C. H., Whang, I., Kim, S. J. \& Lee, J. (2008). Comparative study of two thioredoxin peroxidases from disk abalone (Haliotis discus discus): cloning, recombinant protein purification, characterization of antioxidant activities and expression analysis. Fish Shellfish Immunol 24, 294-307.

Razin, S., Yogev, D. \& Naot, Y. (1998). Molecular biology and pathogenicity of mycoplasmas. Microbiol Mol Biol Rev 62, 1094-1156.

Rhee, S. G., Chae, H. Z. \& Kim, K. (2005). Peroxiredoxins: a historical overview and speculative preview of novel mechanisms and emerging concepts in cell signaling. Free Radic Biol Med 38, 1543-1552.

Rho, B. S., Hung, L. W., Holton, J. M., Vigil, D., Kim, S. I., Park, M. S., Terwilliger, T. C. \& Pedelacq, J. D. (2006). Functional and structural characterization of a thiol peroxidase from Mycobacterium tuberculosis. J Mol Biol 361, 850-863.

Ross, R. F. (1992). Mycoplasmal disease. In Diseases of Swine, pp. 535-549. Edited by B. E. Straw. Ames, IA: Iowa State University Press.

Schafer, E. R., Oneal, M. J., Madsen, M. L. \& Minion, F. C. (2007). Global transcriptional analysis of Mycoplasma hyopneumoniae following exposure to hydrogen peroxide. Microbiology 153, 3785-3790.

Seo, M. S., Kang, S. W., Kim, K., Baines, I. C., Lee, T. H. \& Rhee, S. G. (2000). Identification of a new type of mammalian peroxiredoxin that forms an intramolecular disulfide as a reaction intermediate. J Biol Chem 275, 20346-20354.

Sirand-Pugnet, P., Citti, C., Barre, A. \& Blanchard, A. (2007). Evolution of mollicutes: down a bumpy road with twists and turns. Res Microbiol 158, 754-766.

Stinear, T. P., Seemann, T., Pidot, S., Frigui, W., Reysset, G., Garnier, T., Meurice, G., Simon, D., Bouchier, C. \& other authors (2007). Reductive evolution and niche adaptation inferred from the genome of 
Mycobacterium ulcerans, the causative agent of Buruli ulcer. Genome Res 17, 192-200.

Suttiprapa, S., Loukas, A., Laha, T., Wongkham, S., Kaewkes, S., Gaze, S., Brindley, P. J. \& Sripa, B. (2008). Characterization of the antioxidant enzyme, thioredoxin peroxidase, from the carcinogenic human liver fluke, Opisthorchis viverrini. Mol Biochem Parasitol 160, 116-122.

Tamura, K., Dudley, J., Nei, M. \& Kumar, S. (2007). MEGA4: Molecular Evolutionary Genetics Analysis (MEGA) software version 4.0. Mol Biol Evol 24, 1596-1599.

Vasconcelos, A. T., Ferreira, H. B., Bizarro, C. V., Bonatto, S. L., Carvalho, M. O., Pinto, P. M., Almeida, D. F., Almeida, L. G., Almeida, R. \& other authors (2005). Swine and poultry pathogens: the complete genome sequences of two strains of Mycoplasma hyopneumoniae and a strain of Mycoplasma synoviae. J Bacteriol 187, 5568-5577.

Vilei, E. M. \& Frey, J. (2001). Genetic and biochemical characterization of glycerol uptake in Mycoplasma mycoides subsp. mycoides SC: its impact on $\mathrm{H}_{2} \mathrm{O}_{2}$ production and virulence. Clin Diagn Lab Immunol 8, 85-92.

Wang, Q., Chen, K., Yao, Q., Zhao, Y., Li, Y., Shen, H. \& Mu, R. (2008). Identification and characterization of a novel 1-Cys peroxiredoxin from silkworm, Bombyx mori. Comp Biochem Physiol B Biochem Mol Biol 149, 176-182.

Wood, Z. A., Schroder, E., Robin, H. J. \& Poole, L. B. (2003). Structure, mechanism and regulation of peroxiredoxins. Trends Biochem Sci 28, 32-40.

Edited by: C. Citti 